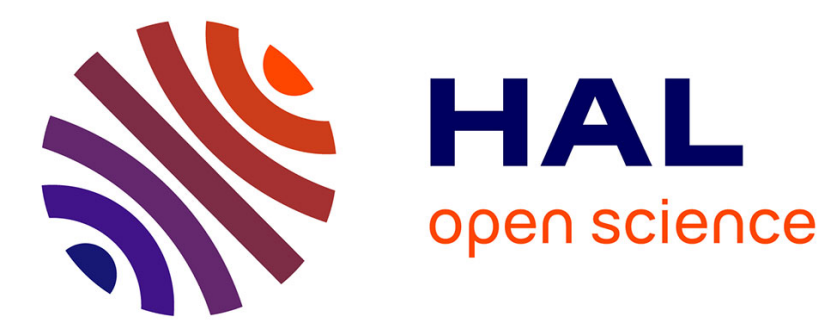

\title{
'It has to become true genetics': tumour genetics and the division of diagnostic labour in the clinic
}

\author{
Claire Beaudevin, Ashveen Peerbaye, Catherine Bourgain
}

\section{To cite this version:}

Claire Beaudevin, Ashveen Peerbaye, Catherine Bourgain. 'It has to become true genetics': tumour genetics and the division of diagnostic labour in the clinic. Sociology of Health and Illness, 2019, 41 (4), pp.643-657. 10.1111/1467-9566.12844 . halshs-02167532

\section{HAL Id: halshs-02167532 \\ https://shs.hal.science/halshs-02167532}

Submitted on 27 Jun 2019

HAL is a multi-disciplinary open access archive for the deposit and dissemination of scientific research documents, whether they are published or not. The documents may come from teaching and research institutions in France or abroad, or from public or private research centers.
L'archive ouverte pluridisciplinaire $\mathbf{H A L}$, est destinée au dépôt et à la diffusion de documents scientifiques de niveau recherche, publiés ou non, émanant des établissements d'enseignement et de recherche français ou étrangers, des laboratoires publics ou privés. 


\title{
'It has to become true genetics' Tumour genetics and the division of diagnostic labour in the clinic Claire Beaudevin ${ }^{1}{ }_{(1)}$, Ashveen Peerbaye ${ }^{2}$ and Catherine Bourgain ${ }^{3}$
}

\author{
${ }^{1}$ CNRS (French National Centre for Scientific Research) / Cermes3 (Centre for research in \\ medicine, science, health, mental health, and society), Villejuif/Paris, France \\ ${ }^{2}$ Paris-Est Marne-la-Vallée University, Laboratoire Interdisciplinaire Sciences Innovations \\ Sociétés (LISIS), Marne-la-Vallée, France \\ ${ }^{3}$ Inserm (French Institute for Medical Research) / Cermes3 (Centre for research in medicine, \\ science, health, mental health, and society), Villejuif/Paris, France
}

\begin{abstract}
Tumour genetics is currently turning into a massive clinical approach. This paper is an enquiry into its practices as they expand beyond expert and experimental contexts and become routinised in clinical hospital settings. Studying a French university hospital, we unpack the content and everyday organization of diagnostic labour in this context. Exploring the sociotechnical frictions that arise in the process, we describe the ways in which they are collectively controlled, and stabilized through organizational fictions, that are instrumental in making tumour genetics doable in the hospital, at a large scale. We further show that the new role of external regulations in the production of clinical values for mutations has a strong impact on diagnostic work, making it possible to be performed locally without resorting to expert bioclinical collectives, and outside the professional jurisdiction of clinical geneticists. This division of labour appears as a necessary condition for the rise in clinical productivity required by a new function assigned to genetics: to guide the prescription of drugs for common diseases. This turn in the way genetics is embedded in the clinic calls for a thorough reassessment of its impacts on clinical discourses, practices and decisions.
\end{abstract}

Keywords: France, Tumour genetics, Biomedical platform, Diagnosis, Clinical genetics, Targeted therapy, INCa, Routine

\section{Introduction}

Oncology has long been a field of interest in social science for studying how 'new genetics' impacts clinical discourses, practices and decisions. Though concerning only a minority of patients affected by cancers, oncogenetics is one of the most active fields for clinical genetic testing, far ahead from the majority of other rare genetic diseases. With the rise of targeted therapies, a new mode of genetic analysis - tumour genetics - has entered the field of oncology. This approach contrasts with oncogenetics on two levels: first, its target of analysis is not an individual's germline DNA, but her tumour's DNA. Second, a mutation is no longer understood as the 
molecularised inscription of a clinical lesion (Foucault 1963, Gaudillière 2002) but as a trace of the tumoural process, signalling a putative response to a particular drug. The types of cancers concerned are numerous - including lung, colorectal, stomach, ovarian, breast cancers, melanoma or leukaemia - and the number of potentially eligible cases is growing. As a result, tumour genetics is turning into a massive practice: in 2016 in France, 83,000 patients had their tumours genetically profiled whereas 38,000 underwent an oncogenetic test.

In this paper, we aim at characterizing the organization of tumour genetics practices as they expand beyond expert and experimental contexts, and become routinised in clinical hospital settings, i.e. institutions with a higher pressure for clinical productivity and a more limited connection to research. As many researchers of technological innovation in healthcare have demonstrated (e.g. Beaudevin and Pordié 2016, Lock et al. 2000), final uses of a technology whether mainstream and compliant or 'diverted' and unsanctioned - are shaped in crucial ways by social and clinical contexts. Routine practices of tumour genetics cannot be understood as mere adaptations of pre-existing arrangements. Rather, they are embedded in a different field of constraints, are organized primarily to meet everyday hospital needs, and are mostly freed from pre-defined technological expectations. Drawing on a collective ethnography, we study the case of a 'tumour molecular genetics platform' set in a French university hospital, where the analyses required for delivering new targeted therapies against metastatic cancers are routinely performed. We unpack the content and everyday organization of the diagnostic labour being performed, describing how actors deal with issues such as: the articulation of DNA sequencers, patient records and kraft envelopes; the practical differences between tumour genetics and 'true genetics'; or the validity of a tumour analysis report.

Our contribution aims at showing that in this routine hospital setting, no 'bioclinical collective' (Rabeharisoa and Bourret 2009) is locally mobilized to perform the clinical work and produce the meaning and relevance of mutations. Because external regulations, including market authorizations and guidelines, define the mutations searched for in the tumours, the diagnostic labour can be further divided, and the diagnoses can be performed outside the professional jurisdiction (Abbott 1988) of clinical geneticists. This reorganization of diagnostic labour, as well as the dynamics of circulation of funds, artefacts and information that characterize the platform make the scaling up of tumour genetics possible: they indeed allow the routine management of significant numbers of genetic analyses by professionals with a more fragmented expertise.

We begin by presenting how the advent of 'new genetics' in the clinic - and especially genomic testing for mutations in tumour cells - has been framed and discussed within social science. This allows us to emphasize the way our research is both linked to, and differs from, the thematic and theoretical interests explored within the sociological literature on 'biomedical platforms'. We then present our case study, and report on its main findings. First, we suggest that tumour genetics - when analysed as a form of molecular medicine being introduced into a hospital's routine does not appear to grow out of the traditional sites and practices of clinical genetics: it emerges in a neighbouring but specific space. Second, we explore the frictions - professional, technological and otherwise - that arise in the process. We show how these frictions are collectively controlled, and stabilized through organizational fictions, that are instrumental in making the routine, large-scale use of tumour genetics in the hospital doable. This in turn participates in constructing a new representation of genetics: faster and relevant to a much larger part of the population.

\section{Biomedical platforms and diagnostic labour in the clinic}

In recent years, an important body of work dealing with the introduction and use of genetic theories and technologies in clinical contexts has emerged. Social scientists have devoted much 
attention to grappling how, and to what extent, 'new genetics' impacts clinical discourses, practices and decisions. In his empirical exploration of the 'geneticisation' thesis propounded by Lippman (1991, 1992), Hedgecoe (2003) describes how, in the context of rare diseases such as cystic fibrosis, genetic explanations become dominant in the clinical space, and play an important role in reconfiguring the classification of diseases and medical practices. However, as has been pointed out by others (see Weiner et al. 2017 for a thorough review), one of the main shortcomings of the 'geneticisation' framework resides in its overly simplistic view of how genetic reductionism is mobilised to legitimate a biologically deterministic understanding of individuals and pathologies. The framework does not pay enough attention to the sociotechnical mediations required for molecular knowledge to affect nosology, diagnosis and care. Conversely, it underestimates the open-ended, uncertain nature of this process, as well as the active role played in it by clinicians, patients, scientists and technicians - resulting in a skewed representation of the complex interplay between genetic diseases and molecular genetics, as well as between genetic testing, categorical work and clinical judgement (Kerr 2004, Latimer 2015, Latimer et al. 2006).

In many ways, Keating and Cambrosio's seminal study of Biomedical Platforms and their subsequent work also challenges this narrative of geneticisation, especially in the field of oncology. Their account of how the cluster of differentiation system was devised and used within immuno-phenotyping platforms as cell-surface markers for the diagnosis of leukaemia is informed by a description of biomedical platforms as 'specific combinations of techniques, instruments, reagents, skills, constituent entities [...], spaces of representations, diagnostic, prognostic, and therapeutic indications, and related etiologic accounts'. More analytically, they define biomedical platforms as 'material and discursive arrangements that act as the bench upon which conventions concerning the biological or normal are connected with conventions concerning the medical or pathological' (Keating and Cambrosio 2003, 4). In this framework, clinical knowledge and practices are at least as crucial to the constitution of new genetics as their biological counterpart. Furthermore, the agency of artefacts and conventions in the production of contemporary biomedicine is foregrounded here, platforms appearing as the crucibles in which novel biomedical entities are forged. This analytical model insists on the pre-eminence of biomedical platforms in the generation, maintenance and transformation of the sociotechnical networks through which coordination is achieved in biomedicine: 'platforms supply networks with conventions, generate novel entities, and entrench them in clinical routines' (Id., 324). The authors argue that this is made possible by intense regulatory work - regulation being understood here in a broad sense, including not only public intervention through regulatory agencies, but also 'de facto, informal, and even tacit agreements, standards, and guidelines' (Id., 258). Regulation therefore includes elements such as categorical work around diagnostic nomenclatures, or the professional and organizational rules that frame activities.

Building upon this framework, Rabeharisoa and Bourret (2009) argue that these forms of regulatory work involving biomedical entities extend far beyond the research laboratory, and permeate clinical activity itself. They suggest that contemporary genetics-inflected clinical practices (as found for example in oncology and psychiatry) are subjected to a three-fold transformation: the emergence of multidisciplinary 'bioclinical collectives' that collaborate across biology and pathology; a change in the content of clinical work, giving rise to what they dub 'clinic of mutations', in which bioclinical collectives 'are led to explore and test the clinical relevance and biological nature of mutations' (Id., 701); and finally, a growing overlap between clinical work and research, since medical judgement and decisions are no longer confined to diagnosis or prognosis, but encompass the 'testing and invention [...] of descriptive and interpretive models that give meaning to mutations and to their complex relations with 
heterogeneous elements that may be involved in the development of [...] diseases' (Id., 709). Their analysis thus reflects that, far from fostering reductionism, 'genetics reinforces the complexity of pathological categories' (Id., 697) and affects the nature of clinical work itself.

More recent scholarship by these researchers and their collaborators draws upon the same analytical framework to characterize the impact of genomic testing in tumour cells on clinical activity. These studies (especially Bourret et al. 2011, Nelson et al. 2013, 2014) reflect on how diagnosis, prognosis and therapeutic decisions are reconfigured by the introduction of sequencing technologies in clinical settings. They consistently highlight three key features: the growing experimentalisation of clinical work, especially through new kinds of clinical trials that link biological evidence to clinical characteristics; the increasingly collective nature of clinical judgement and clinical decision-making; and the role played by different forms of regulatory work in these processes.

Our own investigation is thematically and theoretically related to many of the questions raised and explored by these researchers. We too are interested in understanding the sociotechnical mediations through which tumour genetics becomes integrated into clinical work. However, the focus of our contribution and our perspective are distinct in some important respects. Our analysis is grounded in the ethnography of the clinical work that takes place in the complex inter-organizational setting of a routine hospital platform for molecular genetics of cancer. The quite massive scale of operations, the high degree of standardization, the limited involvement in research that characterize this kind of platform contrast with the more experimental and expert settings that have been heretofore investigated. In our perspective, this calls for careful analysis of the mundane processes through which clinical judgement and decisions might become more distributed in such settings. This includes paying attention to the conflicts, contestations, misunderstandings and negotiation processes through which a collective contour is achieved in the production of diagnosis. In other words, we are less focused on highlighting what is at stake in a new, 'post-genomic' regime of regulation of diagnosis, than in unpacking and describing the content and everyday organization of what counts as diagnostic labour when genomics exits expert contexts and enters more mundane settings with a strong pressure for clinical productivity.

\section{Settings and methods}

In the early 2000s, the European Medicines Agency (EMA) granted the first market authorizations to a new family of cancer drugs, targeting tumours selected on their molecular (mostly genetic) characteristics. In 2006 in France, the National Cancer Institute (INCa) launched a State-funded programme which resulted in the setting up in French university hospitals and cancer centres of 28 'platforms for molecular genetics of cancer' (Nowak et al. 2012). Each platform was required to analyse all the samples collected from both their host institution and neighbouring private pathology labs. The programme included a list of tests to be provided, according to a handful of guidelines (Institut National du Cancer, 2012).

The Institute lacked both the financial and regulatory means to impose and control ways of doing tumour molecular analyses all over the country. It therefore resorted to a policy based on financial incentives to foster local innovation and collaboration. Hospitals were encouraged to set up these platforms drawing on pre-existing technological, professional and organizational resources, at the local level. Not all of these molecular genetic analyses were new, nor did they all rely on cutting-edge technology. Loosely defined in the market authorizations, they consisted in either looking for pre-specified translocations, deletions or amplifications of DNA segments, or in testing for the presence of potential DNA mutations in a pre-defined set of 
genetic coding regions (exons). The INCa also advocated for the certification of routine laboratory practices but did not impose specific processes or technologies for the tasks to be performed. The incentive policy of INCa thus relied on stimulation through the provision of technology and on the assumption that local resources would be idiosyncratically rearranged for making molecular genetics of cancer doable. As such, the scaling up of tumour genetics in France implied organizational bricolage rather than straightforward implementation. This situation has therefore been conducive to the emergence of multiple particular cases of local practices of tumour genetics.

In this paper, we approach the scaling up of tumour genetics in France through the thorough study of one such case. The findings presented here are drawn from a collective ethnographic study conducted as part of a larger project on high-throughput genomics in the clinic. We present and discuss data collected in 2014 and 2015 in one of the INCa 'platforms for molecular genetics of cancer', located in a regional university hospital in South-East France (hereafter 'the Platform'). We conducted in-depth interviews with 10 professionals involved in tumour genetics in the hospital (administrative officers, head of the Platform, anatomopathologists, biologists, etc.) The interviews were open-ended and explored the interviewees' career path, everyday work experience and views on the recent development of their centre and of tumour genetics itself. We also observed the organization of everyday work on the Platform, including activities in the anatomopathology lab, various tasks involving anatomopathologists, molecular biologists and administrative personnel, as well as administrative and clinical staff meetings. Data were organized using Atlas.ti, interview transcripts and observation notes were analysed thematically.

\section{Bricolage on the platform: organizing new circulations within a university hospital}

In this middle-size French university hospital, the trajectory of biological samples on the new Platform is mostly organized between actors previously involved in a variety of biological characterizations of tumours. It all started in 2006, with the drafting of a proposal to the INCa call by two local female doctors, one of them leading a cancer research group. The project involved solid tumours as well as haematological malignancies. One of the authors explains the structure of the proposal:

We had to rely on hospital-based skills, to show that we were already diagnosing, but at the same time [we had to show] that we were strongly rooted in research units. The platform [in the proposal] was centred on three types of tumours: [aside of malignant haemopathies,] there were pulmonary tumours, which was Martine's ${ }^{1}$ [second author of the proposal] activity, and there were cerebral tumours with Prof. Chapatte. - Platform coordinator (MD)

Once granted INCa funds, the team crafted the actual Platform by innovatively organizing the circulations of samples and data. The Platform facilities are hosted by the hospital's Biology and Pathology Centre (hereafter BPC). Two main constraints make the backdrop of the everyday operation on the platform. The first one pertains to topographical and institutional complexity, since five different units, located on four different floors of the BPC building are involved: the department of pathology (which includes a distinct molecular pathology unit), the onco-haematology genetics unit, the biochemistry and biotherapies unit, and the molecular biology facility. Noteworthily, the perimeter of the Platform is far from self-evident for the actors themselves. The second constraint is time-related. Because results are required to prescribe a treatment, most of the time for patients with metastatic cancers, producing them in a 
timely fashion has become a central issue. According to a recent INCa survey (Institut National du Cancer, 2016), the median time lapse between mutation results delivery and prescription is 18 days. This timeframe is to be compared with the several months routinely awaited in clinical genetics.

More than $90 \%$ of the Platform's activity involves processing solid tumour biopsies, in the form of samples fixed in a paraffin block that start and end their journey within the Pathology department. All paraffin blocks, whether sent by external pathology labs, prepared by local anatomopathologists or extracted from the hospital archives, arrive at the desk before being routed for subsequent analyses. The desk secretary is also in charge of preparing and sending the final report to the prescribing clinician and pathology lab. In between these initial and final steps, the sample is diffracted into a handful of slides and digital information. Its characterization may require the intervention of three different units of the BPC for different aspects of tumour analysis. First, the pathology lab may perform a protein immuno-histo-chemistry (IHC) revelation. Second, the molecular pathology lab may proceed with DNA hybridisation (FISH) and third, the molecular biology lab may conduct a DNA mutation analysis.

When IHC is required, technicians prepare slides and reveal them with various antibodies. The coloured slides are read with a microscope by an anatomopathologist. For a characterization of DNA amplification or translocation, other slides are prepared and hybridized with immuno-fluorescent probes. The results are then analysed with a fluorescence microscope by a biologist. For a characterization of the mutation pattern, slides are sent to the molecular biology on the second floor. Technicians extract, quantify and amplify the DNA before sequencing it, using a pyrosequencer. The sequences are then read and analysed by a biologist who validates the pattern of mutations.

In practice, the processing of a tumour biopsy is less linear than it seems. The processes are often conditional to each other: IHC tests will be performed only if the sequencing results warrant it, FISH analyses are only conducted on a subset of samples identified by sequencing and IHC results. The work to triangulate results obtained with different technologies is critical. It helps fostering trust in DNA-based techniques and analyses for actors not directly involved in their conduct. However, it also generates work overload. This is particularly true for the anatomopathologists, potentially creating a bottleneck for the processing of all tumour samples in the hospital.

Sometimes we cannot say whether [the result] is positive or negative, it is inconclusive, it is intermediate, so we are glad to have other techniques that can move the diagnosis forward [...] I think it is really the notion of complementarity that is interesting and it does not cause us so many problems. [But] it takes more work, that's clear. It is an addition to the routine and the work that we had before - Anatomopathologist (MD)

Rather than merely involving DNA sequencing, tumour genetics should thus be considered as a network of heterogeneous and complementary forms of biomolecular analyses, in which DNA sequencing is embedded.

The storage, communication and circulation of a whole variety of other material resources constitute important issues for tumour genetics in this routine hospital setting. People and tools are required both to organize the circulations occurring within, from and towards the Platform and to bring together the different tasks necessary to fulfil the Platform's assignments. This articulation work (Strauss 1985) includes aspects such as managing the stocks of consumables and reagents, or negotiating schedules for equipment use. An eloquent example is the little 'paper technology' (Krajewski 2011) that proved decisive in organizing the circulation of 
objects and information: a simple kraft envelope. As explained by one of the Platform desk secretaries, when she took her position, all the important artefacts were scattered:

When I arrived here [...] the tissue block was on its own, the lab techniques [results] on their own, the patient's file was on its own, everything kept separate. (...) Some things were lost and never recovered, incidentally... The idea behind the kraft envelope is that when a sample arrives with the clinician's prescription, I make a record, and I put everything in the kraft envelope and it stays there, always. - Platform Desk Secretary

This dispersal appeared as a serious issue, endangering the necessary final gathering of slides and results. This envelope is a striking illustration of the seemingly mundane activities and tools that make up articulation work and allow collaboration to take place between the professionals that contribute to the Platform.

Another example is given by the computer information system for data storage and management on the Platform. The pathology department has long been using a different system from that used by other departments. As one of the administrative officers puts it: "[the software applications] don't talk to each other [...] one is like jihadists and the other is Jewish fundamentalists'. Since changing habits or software would have been too costly, the two systems are used in parallel and the desk secretary enters all initial information on samples twice. When needed, she pastes results from one system to the other or to an external file. This manual intervention helps overcoming the disruption in the circulation of the data, but it also creates a notable risk of error: the secretary and the clinician responsible for the analyses must be very watchful over the final results. Here again, articulation work appears as a necessary condition for the smooth functioning of the heterogeneous biomolecular analysis network that makes up tumour genetics on the Platform.

\section{Making tumour genetics: a new technological and epistemic space alongside clinical genetics}

Given the extended presence of clinical genetics, including oncogenetics (Bourret et al. 2006), within French cancer centres and university hospitals, one could have expected these rearrangements to be conducted in close association with local experts in genetics for the clinic. Yet, in the case we investigated, tumour genetics thrives alongside clinical genetics rather than in close cooperation with it. The interactions between germline genetics and tumour genetics mainly consist in the pooling of resources: the fancier, next-generation sequencing (NGS) machines were bought thanks to a national call directed to germline genetics of rare diseases, and an NGS engineer was recruited on a position jointly funded by INCa and the clinical genetics department.

The will [of mutualising] comes from the people here [...] With equipment in the state it was and the needs now, this has become a necessity, because in the NGS era, machines cost a fortune. The purchase of the device is only $10 \%$, roughly, of what has to be done. In order to make a diagnosis in high-throughput genetics, one needs a strong bioinformatics backdrop, servers... So, [mutualising] becomes a necessity. - Head of clinical genetics department ( $\mathrm{PhD})$

Technical and cost-related constraints of NGS rather than clinical expertise were central motivations for this policy, which implied intense negotiations regarding the conditions for sharing data storage, the expertise of the NGS engineer, and access to machines. 
For many health professionals outside the Platform, the existence of a space shared by both tumour and clinical geneticists - also referred to as a 'NGS platform' - contributes to the double impression that 'genetics' is pervasive and ubiquitous; and that the Platform is somehow a further manifestation of traditional clinical genetics. One of the platform professionals explained:

We get this kind of questions: 'we're told about genetics in anatomopathology, in biochemistry, [in obs and gynae, paediatrics], what does it mean? You are all doing the same thing.' - Biologist $(\mathrm{PhD})$

However, despite this blurriness and apparent similarity to untrained eyes, tumour and clinical genetics markedly differ, when it comes to diagnostic labour itself. Contrasting a genetic analysis in tumour genetics (KRAS) and in oncogenetics (BRCA), the Platform coordinator points to a twofold discrepancy:

The big difference is that with KRAS, we search for a mutation at a precise location. It's there or it's not there. It's binary. With BRCA, the mutation can be all over the gene and some have no consequence, they're not even a mutation. Others are deleterious and others are unknown. This means that these mutations must be analysed by the scientists to evaluate their consequences, either in vitro or with segregation analyses in families. There is a whole circuit of work to validate mutations or not. Each family may have a distinct mutation. It is completely different with KRAS, where we search exon 12 for a known mutation. There may be many other mutations in the gene but we don't care. - Platform Coordinator (MD)

First, in many cases, the production of a genetically informed diagnosis of disease (or of disease risk) requires the characterization of an entire gene or gene region, in order to either identify mutations or rule out their presence. As new mutations are regularly described, this search has to be exploratory and often requires a specific expertise on the gene studied. In tumour genetics, as it is performed in the Platform, the molecular characterization follows a different path. All the mutations searched for are part of a pre-defined set. Each and every sample can thus be processed and analysed in the same manner. No expert knowledge of the scrutinized genes is required. Rather, technical dexterity and technology-oriented know-how are needed: the initial steps to extract and prepare the DNA from a sample may involve a particular technical treatment; the final interpretation of the sequencing results may also require precise analytical skills, such as those of a $\mathrm{PhD}$ in biological sciences. This simplification and standardization of the molecular genetic characterization process is essential: it is a crucial condition to handle an increasing number of samples in a much shorter timeframe.

Second, as analysed by Bourret and Rabeharisoa (2008), in clinical genetics, diagnostic work consists in simultaneously producing the clinical relevance and the biological significance of mutations. These two tasks are intrinsically linked. The constitution of large mutation databases, which have become central tools, has not fundamentally changed the nature of diagnostic labour (Timmermans 2015). The triangulation work between the clinic and the biology remains unescapable.

What the type of tumour genetics performed in the Platform accomplishes is to add a further step in the division of diagnostic labour. Work is restricted here to the molecular characterisation of mutations. The evaluation of their effects - i.e. their capacity to guide treatment decision - is not produced locally but taken for granted, resulting from market authorizations issued by the EMA and completed by national directives published by the National Agency for Drug Safety or by the INCa. 
For colon cancer, we used to sequence KRAS exon 2. The market authorization changed last summer [...] Instead of analysing a single exon, we had to analyse six. Organizing such a change in the lab is rather complicated. - Molecular Biologist (PhD)

While insisting on the technical and organizational constraints created by this loss of autonomy, this anecdote illustrates the new dependence on external decision bodies in charge of defining mutation values, for the persons in charge of diagnostic labour. These two activities that make up diagnostic work are therefore uncoupled and conducted by different actors in different places. This transformation, far from being straightforward, is met with resistances and doubts, such as those expressed here:

It [tumour genetics] has to become true genetics. Even if we are not doing germline genetics, it is necessary that germline genetics skills become available within somatic genetic platforms. [...] For me, with advances in NGS, the link with genetics is mandatory because there is a risk with the interpretation of results. [...] Germline and somatic genetics, at some point, have to meet and these molecular genetics platforms, they must really stay genetic.

- Platform Coordinator (MD)

In this vision of future developments, the Platform coordinator criticizes, from both a practical and scientific perspective, the distinction between two types of genetics: one that is deemed able to accommodate a decoupling of diagnostic tasks - i.e. tumour genetics as currently performed on the Platform - and another that cannot - i.e. germline genetics.

\section{Meanings of molecular genetics: $\mathbf{f}(\mathbf{r})$ ictions in the practices of tumour profiling}

The introduction of genomic characterization of tumours in the hospital has undoubtedly contributed to make molecular biology more visible and legitimate in clinical settings. The circulation and use of resources in the Platform - especially INCa funds - play an important role in helping molecular biology gain recognition as a decisive link in the chain of clinical judgement. However, this growing importance is often the source of tensions between professionals, especially between pathologists and molecular biologists. Anatomopathologists, as medical doctors, have historically been key players in the production of cancer diagnosis (Löwy 2010). To this day, it is required that they examine all tumour samples, and they are responsible for signing the final report. The "molecularisation" of diagnosis through sequencing has brought along a few changes in their professional domain, though. For instance, consequently to the creation of the Platform, a molecular biologist with expertise in cancer research, molecular genetics and histology has been recruited within the pathology department. As a "molecular pathologist" who holds a $\mathrm{PhD}$ but is not a medical doctor, she occupies an uneasy professional space, and embodies some of the professional frictions between pathology and molecular biology.

While pathologists advocate for a culture of diagnostic responsibility, molecular biologists lament the shallow understanding of the underlying biological mechanisms among their pathologist colleagues. Both of these views are framed around each other's expertise. Here is a pathologist's view:

In anatomopathology it's not like in biology. Because in biology, outcomes are assays, and assays, and assays. They just click on a button: there is someone, everyday, who is in charge of doing the validation and that's it. So, maybe, there's one [result] that comes out 
red, 'beware, it's damn high', maybe they look at it, but otherwise, no. Whereas we [anatomopathologists], we speak, we express ourselves, in the end there is a diagnosis. We'd better not miss a typo. - Anatomopathologist (MD)

A biologist's perspective makes an interesting counterpoint. She considers molecular biology an important shift in oncology and emphasizes the assets of her own expertise:

Molecular biology is the future of [anatomopathology] [...] Although I'm a molecular biologist and not a medical doctor, I think my diagnosis is more reliable than [that of] a pathologist who is not trained in molecular biology and who won't even look for information about the direction of gene transcription in order to correctly interpret a case. Biologist $(\mathrm{PhD})$

These professional tensions point to different ways of understanding what diagnosis and tumour characterization mean and entail. In the anatomopathologists' perspective, diagnosis belongs to their professional jurisdiction. Through their professional identity, practices and discourse, they manifest an acute awareness of the oftentimes dizzying consequences their diagnosis may have on patients' lives. They therefore embody an attention to the clinical dimension of diagnosis, and feel bound by its consequences. This culture of responsibility oriented towards diagnosis tends to be contrasted with the culture displayed by molecular biologists. The technological and scientific complexity underpinning their practices has become so important - especially as regards the management and interpretation of genomic data - that it is no longer entrusted to technicians alone, and requires extensive biological knowledge and expertise. As a result, molecular biologists are foremost concerned by issues of accuracy and correct interpretation, both in their technological and biological dimensions. Ultimately, these concerns tend to take precedence over the clinical dimension. Novel tumour profiling practices therefore call into question what it means to make a diagnosis, what makes a diagnosis reliable, and what professional responsibility entails.

Besides these professional debates, another type of friction is related to the challenges posed by the introduction of new technologies, protocols and regulations in the Platform's routine operation. We already mentioned the regular updates in the mutations that must be searched for in a given gene. Another good illustration is the switch to 'true' NGS that was slowly being implemented during our fieldwork. As explained by a molecular biologist in charge of the sequencing of tumour samples, the pressure to rapidly adopt new and high-tech machines was confronted with the local needs and means available to support next-generation sequencing activities:

Our routine pyrosequencing technique with the Qiagen [sequencer] is not NGS. [...] It's not sequencing in which we read the whole sequence, we only look for targeted mutations. [We made this choice] because of sensitivity issues, [this sequencer] can detect something as low as $10 \%$ of mutations, which is good for tumour samples containing few tumour cells. We've started NGS, and we've had an [Ion Torrent] PGM for more than a year now. [...] It's very impressive from a technical standpoint, it's a beautiful machine, very sophisticated, but its preparation is very tedious for the technicians. - Molecular Biologist ( $\mathrm{PhD})$

The NGS manager explains that part of her work involves dealing with the potential disruptions brought about by frequent updates in the protocol. These disruptions arise from the instability of these novel technologies, over time and in practice: 
Originally, the protocol would be updated each and every month. Now it's more like every six months. These technologies move a lot. It's what makes them difficult to manage on a day-to-day basis in diagnostics. The machines are new, and prone to technical problems. [...] Updates in the protocol entail changes in the technical procedures, in the machines, in the software for analysis... - NGS Manager (PhD)

As one can imagine, all the modifications that follow updates in the protocol impact the organization of work. Finding the right balance between the array of available sequencing technologies and a robust technological system that gets the job done in a timely and reliable fashion is therefore another dimension of the frictions that surround the practices of tumour profiling. Here, the type of friction involved is akin to Edwards' metaphor of 'data friction', i.e. the costly processes - in time, energy and human attention - that take place at the interfaces between groups, organizations, and machines across which data must move (Edwards 2010, Edwards et al. 2011).

The frictions of the kinds described above exemplify the relative instability of the technologies as well as the plurality of meanings and purposes ascribed to tumour profiling practices in routinised hospital settings. Our observations show that these tensions give rise in return to a series of negotiations and arrangements that ensure the partial integration and alignment of the processes required for the production of a valid diagnosis. The drafting of the final report plays an important role in this regard.

As recommended by the INCa, it consists of a single document to be sent to the prescribing clinician, which combines the results and analyses provided by pathologists and molecular biologists. The results are validated by the professionals who obtained them: the supervising molecular biologist (sequencing results), the molecular pathologist (FISH results), or the anatomopathologist (IHC results). This document therefore contains data obtained through different techniques, provided by different services and travelling through two distinct computer information systems. Depending on the analyses conducted, the report can therefore consist of up to three separate 'parts' and signatures, which are copied and pasted by the Platform desk secretary from each of the two software applications into a single and final electronic document. Far from being straightforward, the finalization of this document is subjected to careful adjustments which reflect various stakes in terms of responsiveness, distribution of expertise and responsibility, and validation processes.

The main issue at stake is the final validating signature, which consists in a mouse click and attributes the responsibility of the entire process to the professional who performs this final click. National regulations currently lead to the anatomopathologists being these professionals, i.e. the ones who are held responsible for the entire diagnosis. The view of one of the molecular biologists emphasizes the stakes of expertise around this report:

What's ludicrous [in the final report process] is that the [anatomopathologists], first don't have time to go back and check everything. And I am the molecular biologist; in this matter, the one who knows how to do [this technique], is me. Therefore I am the one who signs my result. It must then be countersigned by an anapath. But what is the most dangerous? A molecular biologist who is not an anapath but understands the technique and is able to interpret, or an anapath who does not understand anything but simply because he's an anapath, is allowed to sign for a result? - Biologist $(\mathrm{PhD})$

This final report both materializes the professional and technological frictions, and contributes to overcome them, by performing and sustaining a necessary fiction. Indeed, the linear way in which the report is framed, and the formal oversight delegated to anatomopathologists in the 
process meet the social expectations of what diagnosis should be about - i.e. something performed by a medical doctor -, all the while keeping trace of information coming both from histology-based characterization of tumours and molecular tumour profiling. The fiction here is that the diagnosis has been produced by a clinician, according to individualized clinical data and concerns - age, sex, natural history of disease, socioeconomic conditions of the patients, etc. - , while the therapeutic decision ultimately rests on solely molecular criteria.

Another necessary fiction on the Platform pertains to the 'purity' of tumour DNA itself - a target that is almost impossible to achieve in practice, according to the actors. Biopsy samples are indeed never thoroughly tumoural, since the location of the metastases may be difficult to reach and tumour cells are often intertwined with non-tumour cells. This issue is well acknowledged and receives particular attention. The initial task performed by pathologists when a sample enters the Platform consists in evaluating the proportion of tumour cells it contains. Samples with a proportion below $20 \%$ are not supposed to be further analysed. Yet, as noted by one of the Platform's biologists:

We receive many samples below $20 \%$ but we give them a try anyway. If I find a mutation, I report it. If I can't detect any, I say that there is a risk of false negative. For me... I can't say that I won't process a sample. If it was sent to me, it must be that, for this patient, there is no other choice... And we also know that the percentage of tumour cells may vary depending on the pathologist... - Biologist $(\mathrm{PhD})$

Furthermore, no preliminary filter is applied to select only tumour cells when extracting the DNA from a sample. The DNA to be characterized is thus a mixture of germline and tumour DNA. In such a population of DNA sequences, a mutation of interest is not simply present or absent, but detected in a certain proportion of sequences. Its tumoural origin is never questioned but taken for granted. The fiction here is that the DNA analysed is not germinal DNA but a degenerate version of it, sufficiently differing as to not fall under legal regulations. The genetic analyses can thus be conducted outside and beyond the realm of properly authorized geneticists, in labs not fulfilling the standards set by the National Biomedicine Agency to conduct germinal DNA analyses, and without following the information and consent procedure otherwise mandatory.

\section{Discussion and conclusion}

In this paper, we have studied the introduction of tumour genetics in a dedicated platform hosted by a French university hospital. Contrarily to clinical genetics (including oncogenetics) settings, the diagnostic labour performed on the Platform does not primarily rely on categorical work (Latimer et al. 2006) articulating genetic and medical knowledge. Indeed, the meaning of the mutations identified, i.e. the evaluation of their capacity to guide treatment decisions, is not assessed locally but results from external regulatory decisions. No bioclinical collective is locally mobilized to carry out the clinical work and produce the meaning and relevance of mutations.

Yet, what is being performed here is still diagnostic labour, directed towards the production of a treatment decision, based on molecular analyses of tumour samples and performed in a timeframe compatible with urgent care. The issues at stake are more classically related to the organization of work in a complex biomedical context. Indeed, our ethnography shows that tumour genetics on the Platform cannot be reduced to DNA sequencing alone, but is rather embedded in a network of heterogeneous and complementary forms of biomolecular tools and analyses, that mobilizes a variety of specialists, with their own skills and professional cultures. 
This complex sociotechnical network produces important professional and technological frictions, that reveal the plurality of meanings and purposes ascribed to tumour profiling practices in routinised hospital settings. In particular, we show the contrast between molecular biologists and medical pathologists, their perception of what makes a diagnosis reliable and what the locus and focus of their professional responsibility consist in.

We have described the challenges posed by introducing new technologies, protocols and regulations - initially developed in research settings - in routine operations where the priority is to rely on a robust technological system that gets the job done in a timely and reliable fashion. Our study further illustrates the sense of urgency associated with this type of clinical diagnosis. This is shared by all the professionals involved, is tangible in a series of negotiations and arrangements, and fosters the deliberate adoption of different fictions that help overcoming the frictions, in order to perform molecular diagnoses. These fictions concern the nature of the biological material to be analysed (tumour DNA) as well as the meaning of the diagnostic work collectively undertaken.

In her exploration of how different versions of a disease (atherosclerosis) somehow manage to 'hang together' in the hospital, Mol (2002) insists on the importance and relevance of various modes of coordination. They include the circulation of paperwork, the addition and calibration of diagnostic test outcomes, the representation of numbers and data through formulae and pictures, and the pluridisciplinary meetings where specialists collectively discuss the diagnosis and treatment of individual cases (Mol 2002, 84). In her view, these practices, and the way they sustain 'coherence-in-tension' (Mol 2002, 84), partake largely in making clinical work doable. The use of fictions we describe in this paper resonates with this understanding of coordination processes in the hospital - with the additional challenge that what must be made to 'hang together' here, are clinical and biological enactments of cancer. However, these fictions do not simply function as an additional mode of coordination. Rather, we see them as a way of negotiating the meaning and legitimacy of existing work practices (such as drawing together different diagnostic tests into a single report), the authority of those who perform them (pathologists, clinicians, medical biologists, technicians and so on) and the kinds of knowledge they use. These fictions are therefore arrangements that distribute and order different professional and epistemic rationalities in order to make tumour genetics doable.

We do not mean to suggest here that the form of diagnostic labour observed in the Platform, not primarily relying on categorical work, is an intrinsic property of tumour genetics. Studies have precisely discussed the sociotechnical regime associated with tumour genetics, in which mutations have become obligatory passage points (Latour 1988) for the prescription of a given drug to patients, and are referred to as 'actionable' by both oncologists (Carr et al. 2016) and analysts (Cambrosio et al. 2014, Nelson et al. 2013). Relying on detailed descriptions of experimental settings, mainly clinical trials, these studies have shown how the work performed by bioclinical collectives in which clinicians, biologists, bio-informaticians and bio-statisticians make up hypotheses regarding the values of mutations, constitutes a particular mode of drugoriented categorical work.

The point we wish to make here is that in routine hospital contexts, the diagnostic labour associated with tumour genetics can be notably different from that observed in experimental or expert settings. First, the particular organization of the drug market with its strong institutional regulation has introduced the possibility, and potentially the necessity, for a temporal disarticulation between the two tasks required for diagnostic work in genetics. The division of labour between molecular genetics characterization and its clinical implications is taken a step further. Fixed by market authorizations, the clinical value of a mutation is no longer produced locally by bioclinical collectives. Second, far from being an anomaly, this new division of labour appears as a driving force allowing the rise in clinical productivity required by this new 
function assigned to genetics; namely, to guide the prescription of drugs for common diseases. The resulting work required to make a diagnosis can indeed be performed without resorting to expert bioclinical collectives, and outside the professional jurisdiction of clinical geneticists (experts in 'true genetics'). Provided the organization of adequate ways of distributing and recollecting the different tasks, of circulating artefacts, information or financial resources, significant flows of genetic analyses can routinely be managed by professionals with more fragmented but complementary expertise.

We believe that this turn in the way genetics is embedded in the clinic should be considered seriously, given its rising importance. With the regulation of uncertainty no longer performed locally but largely delegated to other regulatory levels (market authorizations, guidelines or technical standards), this new and yet massive context of use calls for a thorough reassessment of the impacts of genomics on clinical discourses, practices and decisions.

Address for correspondence: Claire Beaudevin, Cermes3, campus CNRS, 7 rue Guy Môquet, Villejuif 94801, France.

E-mail: claire.beaudevin@cnrs.fr

\section{Acknowledgements}

We thank the professionals who participated in this research and welcomed us in their labs and offices. We thank the two anonymous reviewers, as well as Ilana Löwy and Jean-Paul Gaudillière for their stimulating comments and suggestions. We also thank Simone Bateman and Dominique Stoppa-Lyonnet, organizers of the 2016 SIRIC Workshop 'From Gene-Targeted Tests to Genome-Wide Data in Cancer Genetics' (Institut Curie, Paris), where we presented a first version of this paper. This research was supported by an exploratory project grant from the Institute for Research and Innovation in Society (IFRIS, Paris, France).

\section{Note}

1 All names are pseudonyms.

\section{References}

Abbott, A. (1988) The System of Professions: An Essay on the Division of Expert Labor. Chicago: University of Chicago Press.

Beaudevin, C. and Pordié, L. (2016) Introduction: diversion and globalization in biomedical technologies, Medical Anthropology, 35, 1.

Bourret, P. and Rabeharisoa, V. (2008) Décision et jugement médicaux en situation de forte incertitude : l'exemple de deux pratiques cliniques à l'épreuve de la génétique, Sciences Sociales et Santé, 26, 33-66.

Bourret, P., Mogoutov, A., Julian-Reynier, C. and Cambrosio, A. (2006) A new clinical collective for French cancer genetics: a heterogeneous mapping analysis, Science, Technology \& Human Values, 31, 431-64.

Bourret, P., Keating, P. and Cambrosio, A. (2011) Regulating diagnosis in post-genomic medicine: re-aligning clinical judgment?, Social Science \& Medicine, 73, 6, 816-24.

Cambrosio, A., Keating, P. and Nelson, N. (2014) Régimes thérapeutiques et dispositifs de preuve en oncologie : l'organisation des essais cliniques, des groupes coopérateurs aux consortiums de recherche, Sciences sociales et santé, 32, 3, 13-42. 
Carr, T.H., McEwen, R., Dougherty, B., Johnson, J.H., et al. (2016) Defining actionable mutations for oncology therapeutic development, Nature Reviews Cancer, 16, 5, 319-29.

Edwards, P. (2010) A Vast Machine: Computer Models, Climate Data and the Politics of Global Warming. Cambridge: MIT Press.

Edwards, P., Mayernik, M.S., Batcheller, A.L., Bowker, G.C., et al. (2011) Science friction: data, metadata, and collaboration, Social Studies of Science, 41, 5.

Foucault, M. (1963) Naissance de la clinique. Une archéologie du regard médical. Paris: Presses Universitaires de France.

Gaudillière, J.-P. (2002) Inventer la biomédecine. La France, l'Amérique et la production des savoirs du vivant (1945-1965). Paris: La Découverte.

Hedgecoe, A. (2003) Expansion and uncertainty: cystic fibrosis, classification and genetics, Sociology of Health \& Illness, 25, 1, 50-70.

Institut National du Cancer (2012) Modèle de compte-rendu de génétique moléculaire pour la recherche de mutations somatiques dans les tumeurs solides Plan Cancer, mesure 21, Soin et vie des malades. Paris: INCa.

Institut National du Cancer (2016) Accès aux tests moléculaires EGFR, RAS ET BRAF. Résultats d'une enquête dans 5 régions françaises Appui à la décision/Recherche.

Keating, P. and Cambrosio, A. (2003) Biomedical Platforms. Realigning the Normal and the Pathological in Late-Twentieth-Century Medicine. Cambridge/London: The MIT Press.

Kerr, A. (2004) Genetics and Society. A Sociology of Disease. Londres: Routledge.

Krajewski, M. (2011) Paper Machines. About Cards \& Catalogs, pp. 1548-929. Cambridge, MA: The MIT Press.

Latimer, J. (2015) The Gene, the Clinic and the Family: Diagnosing Dysmorphology: Reviving Medical Dominance, London: Routledge.

Latimer, J., Featherstone, K., Atkinson, P., Clarke, A., et al. (2006) Rebirthing the clinic: the interaction of clinical judgment and genetic technology in the production of medical science, Science, Technology, \& Human Values, 31, 5, 599-630.

Latour, B. (1988) The Pasteurization of France. Cambridge, MA: Harvard University Press.

Lippman, A. (1991) Prenatal genetic testing and screening: constructing needs and reinforcing inequities, American Journal of Law and Medicine, 17, 15-50.

Lippman, A. (1992) Led (Astray) by genetic maps: the cartography of the human genome and health care, Social Science \& Medicine, 35, 1469-96.

Lock, M.M., Young, A. and Cambrosio, A. (2000) Living and Working With the New Medical Technologies: Intersections of Inquiry. Cambridge: Cambridge University Press.

Löwy, I. (2010) Preventive Strikes. Women, Precancer, and Prophylactic Surgery. Baltimore: Johns Hopkins University Press.

Mol, A. (2002) The Body Multiple: Ontology in Medical Practice. Durham and London: Duke University Press.

Nelson, N.C., Keating, P. and Cambrosio, A. (2013) On being "actionable": clinical sequencing and the emerging contours of a regime of genomic medicine in oncology, New Genetics and Society, 32, 4, $405-28$.

Nelson, N.C., Keating, P., Cambrosio, A., Aguilar-Mahecha, A., et al. (2014) Testing devices or experimental systems? Cancer clinical trials take the genomic turn, Social Science \& Medicine, 111, 74-83.

Nowak, F., Soria, J.-C. and Calvo, F. (2012) Tumour molecular profiling for deciding therapy - the French initiative, Nature Reviews Clinical Oncology, 9, 479-86.

Rabeharisoa, V. and Bourret, P. (2009) Staging and weighting evidence in biomedicine: comparing clinical practices in cancer genetics and psychiatric genetics, Social Studies of Science, 39, 5, 691-715.

Strauss, A. (1985) Work and the division of labor, The Sociological Quarterly, 26, 1, 1-19.

Timmermans, S. (2015) Trust in standards: transitioning clinical exome sequencing from bench to bedside, Social Studies of Science, 45, 1, 77-99.

Weiner, K., Martin, P., Richards, M. and Tutton, R. (2017) Have we seen the geneticisation of society? Expectations and evidence, Sociology of Health \& Illness, 39, 7, 989-1004. 